idea of migratory movements upon a broad front. The extraordinary complexity and variety of migration in different species, sometimes closely related, were illustrated by a series of well-chosen examples, some selected from the recently investigated movements of birds confined to tropical areas; but it was pointed out that overriding all the complexities a regularity of time and place and route characterises migratory movements as a whole. The outstanding problem still to be solved is that of route-finding, and although there are many indications that vision plays an important part (for example, fog is one of the serious disturbances of a migration) yet the orientation of a migration journey must still be looked upon as something of a mystery.

Some interesting results of marking experiments were described by Dr. E. S. Russell in discussing the migrations of fishes, where knowledge of migration is of great economic importance since the movements of concentrated shoals of food fishes regulate the fisheries. In fish-marking experiments, hundreds of thousands of individuals have been marked and a high return of $30-40$ per cent gave a clear indication of the major movements, which was supplemented by the analysis of fishery statistics. Generally speaking, the migrations of fishes consist of three movementsof mature fish to the spawning grounds, generally upstream, a dispersion after spawning, generally downstream, and local seasonal migrations such as that of the tunny into the North Sea in autumn. Dr. Russell illustrated these facts by describing in detail recent observations upon the movements of cod, plaice and salmon.

The series of general papers on migration was concluded by an account of the migrations of insects by Dr. C. B. Williams. In this case, migration does not necessarily include a return flight, though in the last five years evidence has accumulated that some Lepidoptera do make a return journey. Migration is widespread in the insect world, many instances being known from the groups of Lepidoptera, locusts, dragon-flies and beetles. One of the most remarkable cases is that of the American monarch butterfly which spends the summer so far north as Alaska and winters in the southern States and Mexico, returning to a state of semi-hibernation in the same groups of trees year after year, although one or two generations have passed since the former occupants had traversed the migration route. The extraordinary movements of the world-wide painted lady butterfly were described, and it was shown that insect migration takes place in definite directions, and is independent of the direction of the wind. The understanding of insect migration is complicated by the short life of insects which makes impossible any learning of the route from direct ancestral experience.

The migration symposium was a popular feature of the Section's discussions, and was attended by a very large audience.

\title{
Character and Causes of New Stars
}

$\mathrm{A}^{\mathrm{T}}$ the British Association meeting in Norwich, $A$ a discussion on new stars was opened in Section A (Physics) on September 10 by the Astronomer Royal (Dr. H. Spencer Jones), and other papers were contributed by Mr. J. P. M. Prentice, Prof. F. J. M. Stratton, Prof. W. H. McCrea, Mr. E. G. Williams, and Dr. A. B. Wyse and Mr. R. H. Stoy. The following is a summary of some of the chief points whicb emerged.

A new star may be defined for practical purposes as a star the apparent brightness of which increases within a few days by some five magnitudes or more. The first well-authenticated historical record of a nova is of that observed in 1572 by Tycho Brahe, and since then novæ have been looked for and observed with increasing zeal. This may be illustrated by the fact that, since 1901, forty-two galactic novæ have been recorded, and of these seven have been near enough and discovered soon enough for detailed spectroscopic study. These seven include Nova Herculis 1934, discovered by
Prentice, through whose promptitude in communicating the fact a spectrum was obtained by Martin at Greenwich within one hour after discovery.

The 'light-curve' of a nova in general shows a steep rise to its principal maximum, followed at first by a steep fall, and then by a more gradual fading, with small fluctuations, to something very near the pre-outburst magnitude. The case of Nova Herculis, however, showed novel features in the way of many comparable maxima, extending over some months, before the steep drop occurred. The intrinsic surface brightness, as judged by the effective temperature, does not in general change much before the principal maximum, and hence the increase in total brightness must be attributed to an increase in the radiating surface. In a typical case, the radius of the star, as defined by its photosphere, must increase about a hundredfold. In the later stages, however, the effective temperature does increase very considerably, so 
that the final radius when the star has returned to approximately its initial total brightness may be perhaps $1 / 25$ of the pre-outburst radius. The net consequence of the outburst being thus a contraction of the whole star, it results in the liberation of a vast amount of gravitational energy of which some, at any rate, must supply the energy for the increased radiation during the eruption.

The variation of brightness of a nova is accompanied by a highly complex sequence of changes in its spectrum. Before the principal maximum the spectrum is not greatly different from one of the ordinary spectral types. The absorption lines, which are then its chief feature, show by their displacement towards the violet the general swelling up of the star already deduced from its increase in brightness. After maximum, multiple absorptions appear with large violet displacements, and each in general bordered on the redward side by bands of emission the mean position of which coincides in each case with the undisplaced position of the corresponding spectral line. These features can be explained by supposing that one or more shells of gas are thrown off from the star and accelerated away from it, probably under forces due to radiation pressure. This has in some cases been confirmed by the fact that, after enough time has elapsed for these shells to attain sufficient size, it has been possible to observe them directly as a growing nebulosity around the parent star. There is also spectroscopic evidence in some cases for the ejection of matter in irregular jets rather than, or as well as, in symmetrical shells. In addition, turbulence appears to develop in the ejected matter.

In the further spectral development the absorptions gradually disappear, giving way to a bright line spectrum in which a number of 'forbidden' lines of oxygen $[\mathrm{O} \mathrm{I}]$ and $[\mathrm{O} \mathrm{III]}$, and iron [Fe II], make their appearance. The whole sequence is consistent with what may be expected from the increase of temperature of the parent star and the decrease in density of the expanding envelope. The final spectrum is of Wolf-Rayet type, the structure of the lines suggesting the continued emission of gas. It seems probable that the nova is then approaching a white dwarf state.

Photometric measurements of novæ spectra have recently yielded valuable information confirming both the hypothesis of the expanding gaseous shells and also the high colour temperature attained by the nuclear star in the later stages. Further, the photometry of the absorption lines due to interstellar $\mathrm{Ca}^{+}$yields an estimate of the distance of the nova. This method gave for Nova Herculis a distance of 1,200 light-years, which shows that at maximum it was 30,000 times as bright as the sun.

The estimated rate of appearance of novæ in the Milky Way agrees well with Hubble's estimates of the number appearing in the Andromeda nebula, which may be regarded as a comparable system. If this rate is assumed uniform, it shows that in about $3 \times 10^{9}$ years every star would become a nova. This is approximately equal to the lowest estimate of the age of the stars, so it seems likely that such a flare up may in fact happen to every star. The most plausible explanation of the phenomenon is provided by E. A. Milne's theory, according to which instability sets in at a certain epoch in the evolution of a star, causing it to pass over into a 'collapsed' configuration, with the liberation of a large amount of gravitational energy. The same theory shows that, if the star is originally rotating, the collapse may cause it to split into two components, which need not then remain collapsed, thus suggesting an origin for double stars.

It is extremely doubtful whether novæ constitute a homogeneous class of objects. Whereas the total brightness in most cases increases tenor a hundred-thousand fold, it seems that the nova of 1572 underwent an increase of about 16 million fold. Similar cases have been observed in other galaxies. So the isolation of a class of super-novæ is indicated, and it has been suggested by Baade and Zwicky that their outbursts provide the source of cosmic radiation. Again the behaviour of stars like RS Ophiuchi and T Pyxidis makes it seem probable that there is also a class of recurrent novæ.

W. H. MoC.

\section{Geological Relations of Early Man in East Anglia}

$\mathrm{T}$ HE discussion on this topic, held on September 5 at the Norwich meeting of the British Association, has revealed a far greater measure of agreement between geologists and archæologists than has emerged during any previous review of the subject. The division of the East Anglian glacial deposits into four distinct groups has pro- vided a framework into which most of the archæological finds appear to fit quite naturally ; it is thus not surprising that discussion should now centre around the more obvious of the remaining doubts and difficulties.

The first of these concerns the authenticity and cultural position of the various sub-Crag industries. 